\title{
Structure of Liquid GaSb under Pressure
}

\author{
Takanori HATTORI, Naohito TAGA, Yukinobu TAKASUGI, Tetsuji MORI, \\ Kazuhiko TSUJI * \\ Keio Univ., Hiyoshi, Kohoku-ku, Yokohama 223-8522, Japan
}

\section{Introduction}

$\mathrm{GaSb}$ is one of the most popular III-V semiconductors. The structural and electronic properties are similar to those of IV semiconductors. The III-V semiconductors have the ionic characters in chemical bonding compared to IV semiconductors. For the purpose of elucidating the effects of the ionicity in chemical bonding on the pressure-induced structural change of the liquid, we have investigated the structure of the liquid $\mathrm{GaSb}$ up to 9.0 $\mathrm{GPa}$.

\section{Experimental}

The X-ray diffraction patterns were taken by the energy-dispersive method using the synchrotron radiation source and the multi-anvil cell high-pressure apparatus. The pressure and temperature conditions of the measurements are shown in Fig. 1. The data were taken by MAXIII installed at BL-14C2. The effect of the absorption of X-ray by the sample was corrected.

\section{Results and Discussion}

The structure factors, $\mathrm{S}(\mathrm{Q})$ at high pressures are shown in Fig.2. With increasing pressure, the first and second peaks shift toward lower and higher $Q$ values, respectively. It shows that the local structure in liquid $\mathrm{GaSb}$ is not compressed uniformly. The ratio of first and second peak positions, Q2/Q1, approaches to that in liquid Sn with increasing pressure (Fig. 3), which shows that the local structure of liquid $\mathrm{GaSb}$ approaches to that of liquid Sn. The pair distribution functions, $g(r)$ at high pressures are shown in Fig. 4. The position of first peak is almost constant with increasing pressure, while those of hump and second peak decrease. It is considered from the peak positions in high-pressure crystalline form of $\mathrm{GaSb}$ that the local structure of liquid is composed of beta-tin like structure. Based on this model, we found that the liquid $\mathrm{GaSb}$ is compressed by vending of the chemical bond, rather than by the shrink of the chemical bonds.

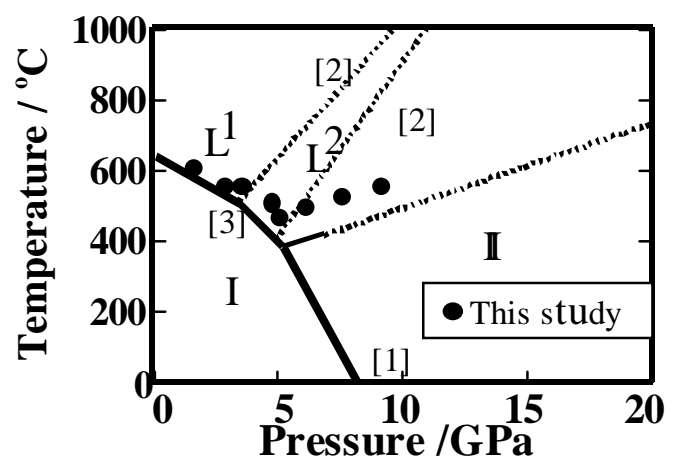

Fig.1 PT conditions for the data collection.

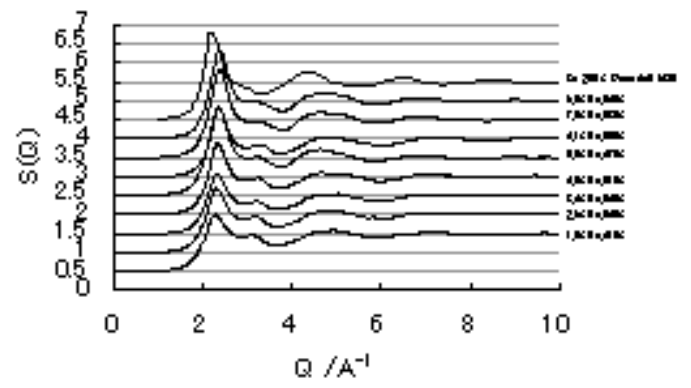

Fig. $2 \mathrm{~S}(\mathrm{Q})$ of liquid $\mathrm{GaSb}$ at high pressures.

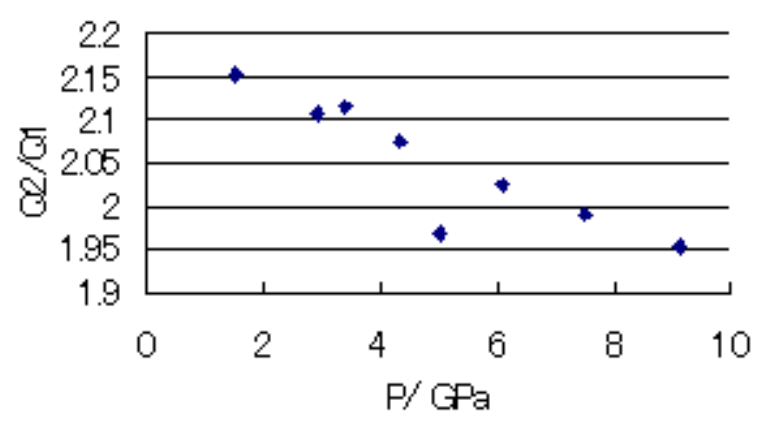

Fig. 3 Q2/Q1 of liquid GaSb at high pressures

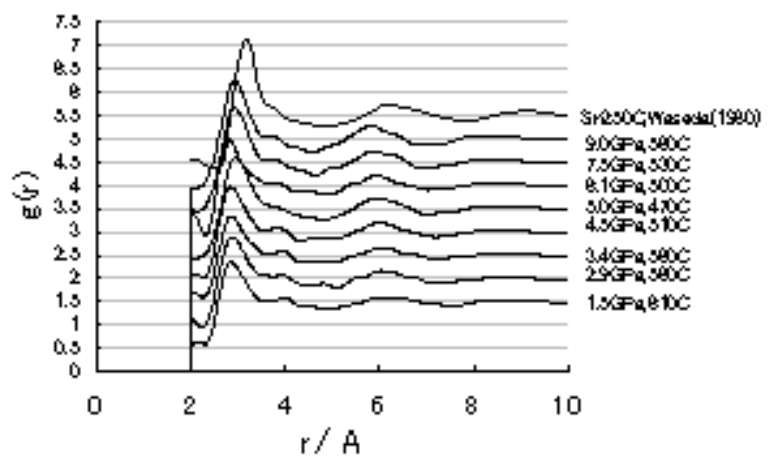

Fig. $4 \mathrm{~g}(\mathrm{r})$ of liquid $\mathrm{GaSb}$ at high pressures.

\section{References}

[1] A. Jayaraman et al., Phys. Rev. 130, 540 (1963).

[2] A. G. Umnov, J. Phys. Condens. Matter. 6, 4625 (1994).

[3] Martinez-Garcia et al., Phys. Stat. Sol. (b) 211, 475 (1999)

[4] Y. Waseda et al., The structure of Non-crystalline Materials- Liquid and Amorphous Solids (McGraw-Hill, New York, 1981)

*tsuji@phys.keio.ac.jp 